\title{
Serologic Detection of Toxoplasma gondii in Cat Owners Residing at Dhaka Metropolitan Area of Bangladesh
}

\author{
Jerin-E-Gulshan*, Niladri Paul*, Atiqur Rahman, Taibur Rahman\# \\ Laboratory of Infection Biology, Department of Biochemistry and Molecular Biology, University of Dhaka, Dhaka, Bangladesh \\ Email: "taibur@du.ac.bd
}

How to cite this paper: Gulshan, J.E., Paul, N., Rahman, A. and Rahman, T. (2021) Serologic Detection of Toxoplasma gondii in Cat Owners Residing at Dhaka Metropolitan Area of Bangladesh. Advances in Microbiology, 11, 257-265.

https://doi.org/10.4236/aim.2021.115019

Received: March 25, 2021

Accepted: May 15, 2021

Published: May 25, 2021

Copyright (C) 2021 by author(s) and Scientific Research Publishing Inc. This work is licensed under the Creative Commons Attribution International License (CC BY 4.0).

http://creativecommons.org/licenses/by/4.0/

\begin{abstract}
Toxoplasma gondii is a zoonotic protozoan that can infect any warm-blooded mammal. T. gondii infects about one-third of the human population on the planet. Infection with the parasite in human causes toxoplasmosis that may pose a high risk in immunocompromised individuals under certain clinical conditions. Cats are the ultimate hosts of $T$. gondii where oocysts are formed through mating of male and female gametes. Infected cats can expel $T$. gondii oocysts in their feces, and thereby capable of pass on a disease to humans and other animals through consumption of foods, vegetables and water that are polluted with cat feces. The study was conducted to detect the presence of anti- $T$. gondii IgM and IgG antibodies in the blood of individuals with or without cat contact to determine if there is any relationship between cat contacts and T. gondii infection in humans. To address this, we enrolled subjects who contacted with the cat as target group and individuals with no cat contact as control group. Following register of different demographic data (including age, sex, education, foods habit, income status, etc.), whole blood from each enrolled subject of both the target group and control group was collected for serum preparation. $T$. gondii infected subjects were detected by Toxo Rapid test kit through identifying anti- $T$. gondii IgM and IgG antibodies in their serum. We found that only three out of twenty subjects who were in contact with cat showed positive IgG response while IgM antibody response was absent for all subjects. When compared with the data from control group, we did not find any significant association $(\mathrm{p}=0.33)$ of cat contact with the transmission of $T$. gondii into human. However, with this small number of study subjects, we cannot conclusively say that there is no impact of cat contact on the transmission of $T$. gondii into human. Whether any association exists or not can be ascertained with a large number of subjects from different areas of Bangladesh in a future study in the population.
\end{abstract}




\section{Keywords}

Toxoplasma gondii, Cat Owner, Seropositivity, Transmission

\section{Introduction}

Toxoplasmosis is a disease caused by Toxoplasma gondii ( $T$. gondii), an obligate intracellular zoonotic protozoan parasite that can infect any warm-blooded animal, including humans. T. gondii specific antibodies are found in the blood of approximately 30 - 50 percent of the world's population [1] [2]. The cat is the parasite's definitive host, where it reproduces sexually [3]. After fusion of male and female gamete in cat intestine, oocysts are released in the environment through cat feces [4]. These oocysts are stable to heat and other unfavorable conditions, and thus continue the environment for an extended time [5]. T. gondii oocysts can contaminate foods [6], vegetables [7] and water [8] that are haunted by the host as an example livestock animals and humans [9]. After ingestion of the oocysts, fast replicating tachyzoites develops which causes an acute infection. During acute infection, this parasite undergoes stage conversion from its fast replicating tachyzoites into slow replicating dormant bradyzoites preferentially within the brain and striated muscle cells and are liable for establishing lifelong persistent infection [10]. T. gondii acute infection is relatively mild and self-limiting with flu-like symptoms in immunocompetent hosts [11]. Pregnant women are at risk because the parasite can spread to the developing fetus, causing irregular brain development or even death of the fetus [12] [13]. T. gondii infection may develop severe neurodegenerative diseases after reactivation in immunocompromised patients [14] [15], or it can disseminate into the eye to cause ocular diseases i.e. blindness [15].

In USA, the rate of chronic $T$. gondii infection in pregnant women characterized by the presence of IgG antibodies is $45 \%$ which is lowest as compared to Europe $~ 65 \%$ [16] [17]. T. gondii was found to be present in approximately $33 \%$ of pregnant women around the world [17]. In addition, approximately 5000 new cases of congenital toxoplasmosis are estimated each year that results in abnormal neonatal effects, including developmental delay, premature death etc. [18]. Although multiple transmission routes are there it was reported that $27 \%$ cat was identified as a carrier of $T$. gondii in a study [19] [20]. The prevalence of $T$. gondii infection varies from country to country. Australia had the highest rate of $T$. gondii infection in domestic cats (52\%), while Asia had the lowest rate (27\%) [20].

Infected cats can shed oocysts upto 4 weeks after getting the primary infection. Therefore, cats act as direct source of $T$. gondii transmission by releasing oocysts into environment which can be taken up by human through contaminated foods, water and vegetables.

In Bangladesh, the frequency of T. gondii infection varies from $16 \%-40 \%$ in humans and upto $70 \%$ in livestock animals as reviewed by Rahman and col- 
leagues [21]. However, there is no data available on how the parasite can transmit into animal and human hosts. Research on T. gondii in humans and animals is extremely limited in Bangladesh. Since cat feces are potential route of transmission, we hypothesize that individuals who are in contact with cat may have higher chance of acquiring infection with the parasite compared to those who do not have any cat contact. To address this hypothesis, the aim of this study is to find out whether a cat owner has a chronic $T$. gondii infection and to see whether there is a connection between cat touch and $T$. gondii transmission to humans. Our research can include preliminary data on the transmission of $T$. gondiimediated diseases from animals to humans.

\section{Methods}

\subsection{Study Design and Subject Enrollment}

The target population for this study was cat owners, their family members who were direct contact with cats at their home and age-matched control subjects who were not in contact with cat of Dhaka Metropolitan Area. The study was conducted from April 2019 to March 2020. Before enrolling subjects, a prior communication was established at the personal level with the guardian of cat owners and, an agreement was made and written consent was taken to collect blood samples and demographic data from the study subjects, in compliance with the rules and regulations for human studies with international standards. The cat owners were interviewed to collect demographic data, including ages, sex, food habits, cat contact severity, etc.

\subsection{Sample Collection}

Three to five milliliter $(3.0-5.0 \mathrm{ml})$ of whole blood was collected from the study subjects of cat owners and their family members $(\mathrm{N}=20)$ and control subjects $(\mathrm{N}=20)$ in commercially available clot activator containing red-topped tubes. Immediately, samples were transported with appropriate measures to the research station at Infection Biology Laboratory, Department of Biochemistry and Molecular Biology, University of Dhaka for the execution of experimental analyses.

\subsection{Separation of Serum}

After collecting whole blood in red-topped tubes, it was left undisturbed at room temperature for 30 minutes to clot. The blood was then centrifuged for $15 \mathrm{mi}$ nutes at $1000-2000 \mathrm{~g}$ in a refrigerated centrifuge. The supernatant was carefully collected by leaving the pellet in the bottom of red-topped tubes and transferring it into a new test tube with the aid of a Pasteur pipette. The resulting supernatant is referred to as serum, and it should be handled at $2^{\circ} \mathrm{C}-8^{\circ} \mathrm{C}$. Since the serum cannot be tested right away, it was divided into $0.5-1.0 \mathrm{ml}$ aliquots, frozen, and transported at $-20^{\circ} \mathrm{C}$. Many serum components are damaged by freeze-thaw cycles, so it's crucial to stop them. 


\subsection{Diagnosis of T. gondii Infected Subjects Using Toxo IgG/IgM RapiCardInsta Test}

The study participants were tested for T. gondii infection using the Toxo IgG/ IgM RapiCardTM InstaTest kit (Cortez Diagnostics, Inc, California, USA) according to the manufacturer's instructions. Toxo IgG/IgM RapiCardTM InstaTest is a horizontal flow chromatographic immunoassay for detecting and distinguishing anti- $T$. gondii IgM and anti- $T$. gondii IgG in human whole blood, serum, or plasma.

Separated serum, buffer, and/or controls were equilibrated with room temperature $\left(15^{\circ} \mathrm{C}-30^{\circ} \mathrm{C}\right)$ prior to performing the examination. The test cassette was removed from the sealed pouch and used within one hour since, according to the manufacturer's advice, the best results are obtained when the assay is conducted as soon as possible. The test cassette was set up on a clean, flat surface. After that, the dropper was then held vertically, the specimen was pulled about $1 \mathrm{~cm}$ above the upper end of the nozzle, and 1.0 maximum drop (approximately 20L) was transferred to each sample well, followed by 2.0 drops of buffer. The test cassette was incubated for 15 - 20 minutes after adding the serum and buffer to form colored lines. The existence of a colored line in the control line region $(\mathrm{C})$ and one or two colored lines in the respective IgM and/or IgG region (s) were used to interpret positive results.

\subsection{Data Analysis}

Statistical analyses and preparation of figures and tables were carried out using Microsoft excel. Association of cat contact and acquiring infection with $T$. gondii was determined using student t-test.

\section{Results}

\subsection{Demographic Data of Study Subjects and Control Subjects}

Individuals contacting of cats at their home with varying degrees of exposure were enrolled in this study. A total of twenty subjects (both male and female) who were in contact with cats and the equal number of control subjects (without cat contacts) were enrolled in the study. Before collecting blood, demographic data such as age, sex, education, occupation, blood type, food habit, and cat contacts were gathered. The subjects in this study ranged in age from 15 to 70 years old. The demographic characteristics of study and control subjects are shown in Table 1.

\subsection{Demographic Variable Associated with Study Subjects}

This study was designed for targeting the population who had contact with cats at their home. The demographic distribution was as follows. Study subjects showed $55 \%$ male and $45 \%$ female subject (Figure 1(a)) where they were exposed to cats with different severity. Figure 1 (b) shows that $40 \%$ of individuals had high contact, $25 \%$ had moderate and $35 \%$ had very low contact with the cat 
at home. Contact with cats is important as this might increase the possibility of T. gondii transmission into human subjects. Among the study subjects, majority had the blood group $\mathrm{A}^{+}(12)$ and $\mathrm{O}^{+}(4)$, whereas blood group $\mathrm{B}^{+}$and $\mathrm{AB}^{+}$shared an equal number of 2 (Figure $1(c)$ ).

Table 1. Demographic data of study and control subjects.

\begin{tabular}{ccc}
\hline Characteristics & Cat Contacts & Control Subjects \\
\hline Study participants (Number) & $\mathrm{N}(20)$ & $\mathrm{N}(20)$ \\
Age (Average in Years) & 35 & 35 \\
Sex & Male: 9 & Male: 14 \\
& Female: 11 & Female: 6 \\
Blood Group & $\mathrm{A}^{+}: 12$ & $\mathrm{~A}^{+}: 9$ \\
& $\mathrm{~B}^{+}: 2$ & $\mathrm{~B}^{+}: 4$ \\
& $\mathrm{O}^{+}: 4$ & $\mathrm{O}^{+}: 6$ \\
Education & $\mathrm{AB}^{+}: 2$ & $\mathrm{AB}^{+}: 1$ \\
& Secondary: 5 & Secondary: 4 \\
& College: 3 & College: $2^{2}$ \\
Occupation & University: 12 & University: 14 \\
& Employed: 10 & Employed: 8 \\
Food Habit & House-wife: 5 & House-wife: 4 \\
& Student: 5 & Student: 8
\end{tabular}

(a)

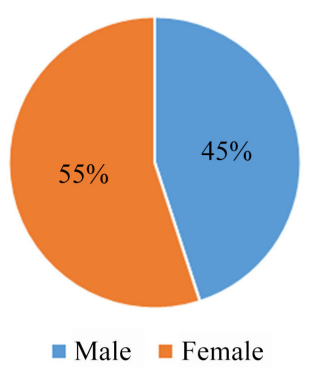

(b)

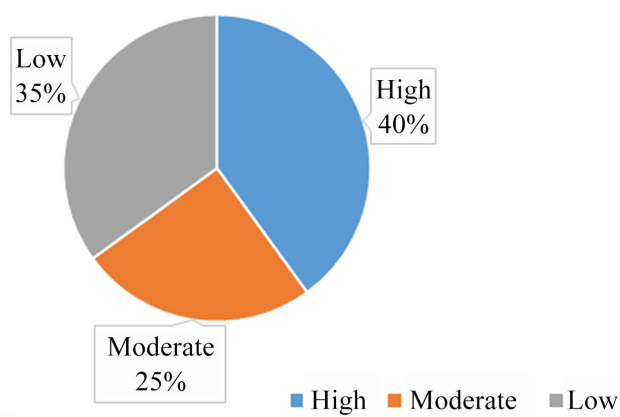

(c)

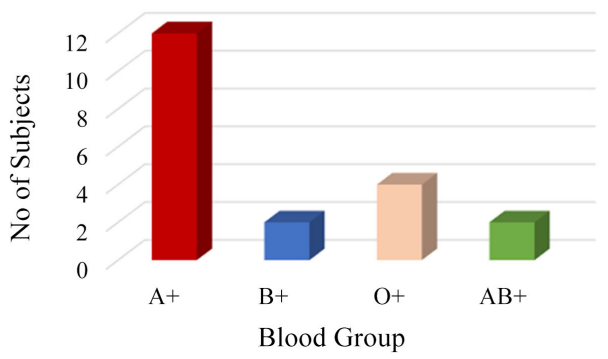

Figure 1. Distribution of demographic characteristics of study subjects who were in cat contacts. (a) Distribution of male and female; (b) Severity of cat contacts (high, medium and low), and (c) Distribution of blood groups among targeted study subjects (those were in contact with cats) are presented. 


\subsection{Detection of T. gondii in Serum of Study Subject with Cat Contacts and Controls}

Anti-Toxoplasma IgM and IgG antibodies were measured in serum of individuals with cat contact and control subjects using rapid test Toxo IgG/IgM RapiCard $^{\text {mox }}$. Our data showed that the level of anti- $T$. gondii IgM was nearly undetectable in serum of both cat contacts and control subjects whereas $\sim 14$ percent study subjects showed positive for anti- $T$. gondii IgG as compared to $9 \%$ in control subjects (Figure 2). However, the increased seropositivity of anti- $T$. gondii IgG in individuals with cat contact was not statistically significant different compared to control subjects ( $\mathrm{p}=0.33$ ) (Figure 2$)$, suggesting that there is no association of cat contact with the transmission of $T$. gondii into the human host, although the finding is limited by the fact that the sample size was very small.

\section{Discussion}

Domestic cat sheds oocysts through feces and is epidemiologically important as it can transmit $T$. gondii into other animals and humans [20]. Consequently, it is essential to investigate the association of cat contacts and $T$. gondii transmission. In some countries, shedding of oocysts was found to be correlated with a high frequency of $T$. gondii infection [20]. In addition, it can cause a sudden outbreak of toxoplasmosis and be identified as a significant cause of infection [22]. For example, it was observed that one single bradyzoite tissue cyst from field cat was enough for producing successful T. gondii infection [3].

In this study, the occurrence of $T$. gondii as determined by the existence of anti-T.gondii IgM and IgG antibodies in serum of subjects who were in contact with cat and control subjects (who were not in contact with cat) has been reported. We established that there was no direct association of cat contacts with

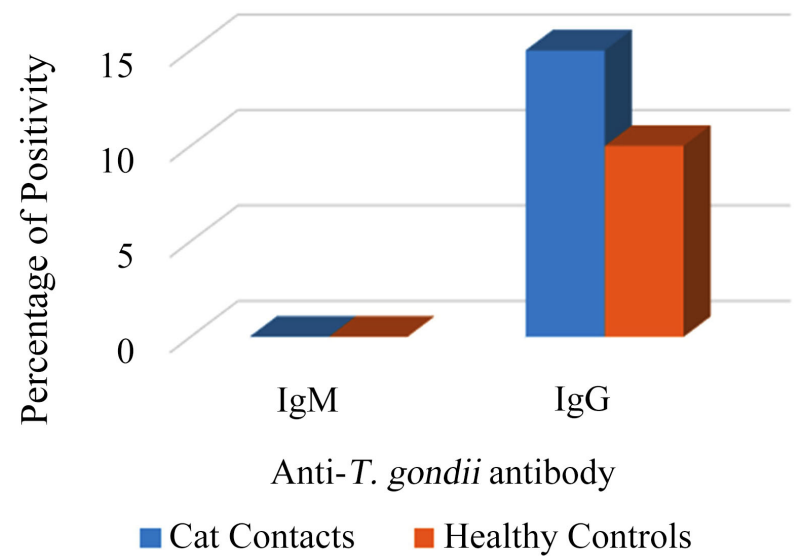

Figure 2. Percentage of anti- $T$. gondii IgM and IgG antibody positivity among study and control subjects. The presence of anti- T. gondii IgM and IgG were determined using rapid test Toxo IgG/IgM RapiCard ${ }^{\mathrm{TM}}$. Percentage comparison of seropositivity of anti- $T$. gondii $\operatorname{IgM}$ and $\operatorname{IgG}$ antibodies between study $(\mathrm{N}=20)$ and control $(\mathrm{N}=20)$ subjects are shown. The Statistically significant difference between was calculated by students-t-test. 
the transmission of $T$. gondii in human subjects. This can be explained in a way that if the cat is not acutely positive for $T$. gondii antibodies, there will be no risk of $T$. gondii transmission into the human host. In addition, cat releases oocysts only within a very short period of time after getting the primary infection. If any subject does not come in contact with cats in the time period, it is unlikely to transmit the parasite. This also suggests that it is necessary to include cats as study subjects along with human subjects to further understanding the possible transmission routes.

T. gondii infection in the feline has been reported to be variable rate $(30 \%$ $52 \%)$ globally. In japan, approximately $5 \%$ of felines were found to be positive for $T$. gondii infection [23], whereas it was significantly higher (87\%) in Ethiopia [24]. This diverse magnitude of infection in cat may increases the possibility of T. gondii transmission into humans. However, in a meta-analysis of cat contact and transmission of $T$. gondii, Huan Ding and colleagues identified two factors (e.g. age and stray or domestic characteristics of cat) are important and significantly associated with $T$. gondii seroprevalence [25]. It was anticipated that the degree of $T$. gondii infection in cat owners or subjects in cat contact would be higher because the cat serves as the definitive host for $T$. gondii reproduction and transmission. Despite our results of no association with cat contacts and anti- $T$. gondii antibodies, we cannot rule out the possibilities of $T$. gondii transmission by this route. Furthermore, cat poop from soil/house hold material can be tested to confirm transmission T. gondii in Bangladeshi population.

\section{Conclusion}

Research on transmission approach of $T$. gondii is vital for understanding the epidemiology of the parasite and to control the parasite at limited hosts. As $T$. gondii is broadly dispersed in natural surroundings and can infect any nucleated cells, therefore it is necessary to determine the likely paths of transmission from its decisive host to other animals and humans. This study would help to maintain the hygiene for the pet animal cat and to reduce the $T$. gondii transmission into other intermediate hosts, including humans of Bangladesh. Although we found no link between cat contact and $T$. gondii infection, a further in-depth analysis would be needed for confirming the role of cat in human transmission. The sample size was limited to twenty for both target and control group which was the major limitation for this study. The reason of small number of sample size is due to the unavailability of new subjects because of emergence of COVID-19 pandemic.

\section{Acknowledgements}

The authors thank Biotechnology Research Center, University of Dhaka for funding the project.

\section{Conflicts of Interest}

The authors declare no conflicts of interest regarding the publication of this paper. 


\section{References}

[1] Pappas, G., Roussos, N. and Falagas, M.E. (2009) Toxoplasmosis Snapshots: Global Status of Toxoplasma gondii Seroprevalence and Implications for Pregnancy and Congenital Toxoplasmosis. International Journal for Parasitology, 39, 1385-1394. https://doi.org/10.1016/j.ijpara.2009.04.003

[2] Flegr, J., Prandota, J., Sovičková, M. and Israili, Z.H. (2014) Toxoplasmosis-A Global Threat. Correlation of Latent Toxoplasmosis with Specific Disease Burden in a Set of 88 Countries. PLoS ONE, 9, e90203. https://doi.org/10.1371/journal.pone.0090203

[3] Dubey, J.P. (2009) History of the Discovery of the Life Cycle of Toxoplasma gondii. International Journal for Parasitology, 39, 877-882. https://doi.org/10.1016/j.ijpara.2009.01.005

[4] Dubey, J.P., Ferreira, L.R., Martins, J. and Jones, J.L. (2011) Sporulation and Survival of Toxoplasma gondii Oocysts in Different Types of Commercial Cat Litter. Journal of Parasitology, 97, 751-754. https://doi.org/10.1645/GE-2774.1

[5] Meerburg, B.G. and Kijlstra, A. (2009) Changing Climate-Changing Pathogens: Toxoplasma gondii in North-Western Europe. Parasitology Research, 105, 17-24. https://doi.org/10.1007/s00436-009-1447-4

[6] Jones, J.L., Dargelas, V., Roberts, J., Press, C., Remington, J.S. and Montoya, J.G. (2009) Risk Factors for Toxoplasma gondii Infection in the United States. Clinical Infectious Diseases, 49, 878-884. https://doi.org/10.1086/605433

[7] Lass, A., Ma, L., Kontogeorgos, I., Zhang, X., Li, X. and Karanis, P. (2019) First Molecular Detection of Toxoplasma gondii in Vegetable Samples in China Using Qualitative, Quantitative Real-Time PCR and Multilocus Genotyping. Scientific Reports, 9, Article No. 17581. https://doi.org/10.1038/s41598-019-54073-6

[8] Gajadhar, A.A., Scandrett, W.B. and Forbes, L.B. (2006) Overview of Food- and Water-Borne Zoonotic Parasites at the Farm Level. Revue scientifique et technique, 25, 595-606. https://doi.org/10.20506/rst.25.2.1679

[9] Jones, J.L. and Dubey, J.P. (2012) Foodborne Toxoplasmosis. Clinical Infectious Diseases, 55, 845-851. https://doi.org/10.1093/cid/cis508

[10] Dubey, J.P., Lindsay, D.S. and Speer, C.A. (1998) Structures of Toxoplasma gondii Tachyzoites, Bradyzoites, and Sporozoites and Biology and Development of Tissue Cysts. Clinical Microbiology Reviews, 11, 267-299. https://doi.org/10.1128/CMR.11.2.267

[11] Taila, A.K., Hingwe, A.S. and Johnson, L.E. (2011) Toxoplasmosis in a Patient Who Was Immunocompetent: A Case Report. Journal of Medical Case Reports, 5, Article No. 16. https://doi.org/10.1186/1752-1947-5-16

[12] Gk, K., Er, N., Saha, A., Habib, A. and Mm, R. (2014) Congenital Toxoplasmosis Associated with Severe Intracerebral Calcification and West Syndrome. Bangladesh Medical Journal, 43, 5-7.

[13] Chen, K.T., Eskild, A., Bresnahan, M., Stray-Pedersen, B., Sher, A. and Jenum, P.A. (2005) Previous Maternal Infection with Toxoplasma gondii and the Risk of Fetal Death. American Journal of Obstetrics and Gynecology, 193, 443-449. https://doi.org/10.1016/j.ajog.2004.12.016

[14] Lee, S.-B. and Lee, T.-G. (2017) Toxoplasmic Encephalitis in Patient with Acquired Immunodeficiency Syndrome. Brain Tumor Research and Treatment, 5, 34-36. https://doi.org/10.14791/btrt.2017.5.1.34

[15] Luft, B.J. and Remington, J.S. (1992) Toxoplasmic Encephalitis in AIDS. Clinical 
Infectious Diseases, 15, 211-222. https://doi.org/10.1093/clinids/15.2.211

[16] Jones, J.L., et al. (2003) Toxoplasmosis-Related Knowledge and Practices among Pregnant Women in the United States. Infectious Diseases in Obstetrics and Gynecology, 11, 139-145. https://doi.org/10.1080/10647440300025512

[17] Bigna, J.J., et al. (2020) Global, Regional, and Country Seroprevalence of Toxoplasma gondii in Pregnant Women: A Systematic Review, Modelling and Meta-Analysis. Scientific Reports, 10, Article No. 12102. https://doi.org/10.1038/s41598-020-69078-9

[18] Jones, J.L., Kruszon-Moran, D., Wilson, M., McQuillan, G., Navin, T. and McAuley, J.B. (2001) Toxoplasma gondii Infection in the United States: Seroprevalence and Risk Factors. American Journal of Epidemiology, 154, 357-365. https://doi.org/10.1093/aje/154.4.357

[19] Hsu, V., Grant, D.C., Zajac, A.M., Witonsky, S.G. and Lindsay, D.S. (2011) Prevalence of IgG Antibodies to Encephalitozoon cuniculi and Toxoplasma gondii in Cats with and without Chronic Kidney Disease from Virginia. Veterinary Parasitology, 176, 23-26. https://doi.org/10.1016/j.vetpar.2010.10.022

[20] Montazeri, M., et al. (2020) The Global Serological Prevalence of Toxoplasma gondii in Felids during the Last Five Decades (1967-2017): A Systematic Review and Meta-Analysis. Parasites and Vectors, 13, 82. https://doi.org/10.1186/s13071-020-3954-1

[21] Rahman, T., Rahman, A. and Chakraborty, S. (2018) Infection of Toxoplasma gondii in Humans and Livestock Animals: An Emerging Silent Threat for Bangladesh. Open Journal of Medical Microbiology, 8, 109-117. https://doi.org/10.4236/ojmm.2018.84010

[22] Tenter, A.M., Heckeroth, A.R. and Weiss, L.M. (2000) Toxoplasma gondii: From Animals to Humans. International Journal for Parasitology, 30, 1217-1258. https://doi.org/10.1016/S0020-7519(00)00124-7

[23] Maruyama, S., et al. (2003) Seroprevalence of Bartonella henselae, Toxoplasma gondii, FIV and FeLV Infections in Domestic Cats in Japan. Microbiology and Immunology, 47, 147-153. https://doi.org/10.1111/j.1348-0421.2003.tb02798.x

[24] Gebremedhin, E.Z. and Tadesse, G. (2015) A Meta-Analysis of the Prevalence of Toxoplasma gondii in Animals and Humans in Ethiopia. Parasites \& Vectors, 8, Article No. 291. https://doi.org/10.1186/s13071-015-0901-7

[25] Ding, H., Gao, Y.-M., Deng, Y., Lamberton, P.H.L. and Lu, D.-B. (2017) A Systematic Review and Meta-Analysis of the Seroprevalence of Toxoplasma gondii in Cats in Mainland China. Parasites \& Vectors, 10, Article No. 27.

https://doi.org/10.1186/s13071-017-1970-6 